\title{
Characterisation of apo E knockout mice as an animal model for liver proteome analysis of Non-alcoholic fatty liver disease
}

\author{
C. Spanos, K. Arnold, E. Oviedo-Orta and J. B. Moore \\ Faculty of Health and Medical Sciences, University of Surrey, Guildford GU2 7XH, UK
}

Non-alcoholic fatty liver disease (NAFLD) is now the most common liver disease worldwide. The strong association between NAFLD, the metabolic syndrome and CVD has raised the question of whether or not NAFLD plays a role in the development or progression of CVD. ${ }^{(1)}$ In particular, while Apo E knockout mice $\left(\mathrm{apoE}^{-/-}\right)$, an animal model that has been extensively used for atherosclerosis research, have been shown to produce features of NAFLD when subjected to high-fat diet (HFD) ${ }^{(2)}$, it is not known whether or not this occurs before, after or concomitant to vascular plaque development.

The objectives of these experiments were to fully characterise the timeline of fatty liver and plaque development in apoE $\mathrm{E}^{-1-}$ animals prior to liver proteome analysis. apoE ${ }^{-1-}$ and C57BL/6, the background strain control, mice were fed for 4, 8, 12 and 16 weeks $(n 3$ from each strain for each time point) either normal chow (NC) or HFD. Extensive liver histology was done at each time point including: haematoxylin and eosin staining to assess liver architecture; Masson's trichrome staining to assess fibrosis; and Oil Red-O staining to assess fat accumulation. In addition, haematoxylin and eosin staining of the brachiocephalic artery and aortic root was used to monitor atherosclerotic plaque development. Plasma lipids, including TAG, total cholesterol (TC), HDL and LDL were also measured at each time point.
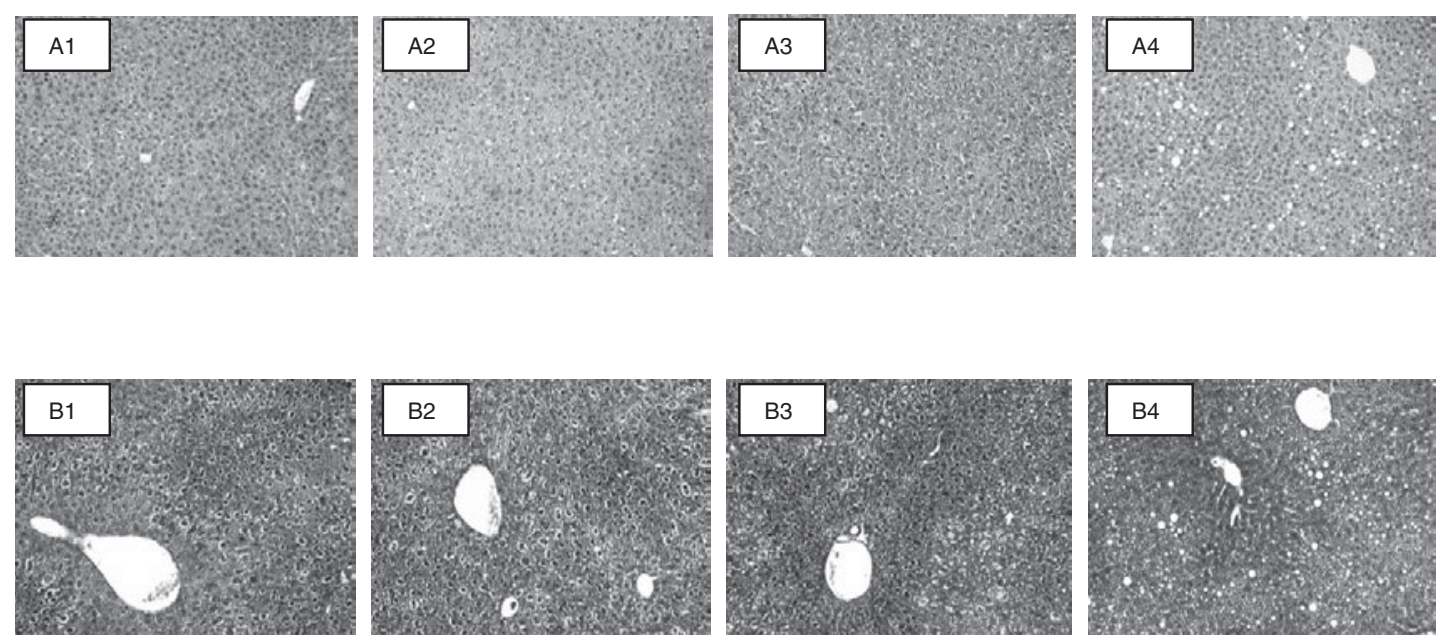

Fig. 1. Liver histology of mice sacrificed after 12 weeks of feeding either normal chow (NC) or high fat diet (HFD). A. haematoxylin and eosin staining of C57BL/6-NC (A1),

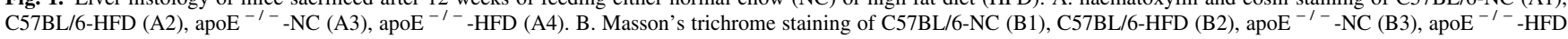
(B4).

Liver haematoxylin and eosin sections showed pronounced macrovesicular steatosis and signs of inflammation in the apoE ${ }^{-/-}-\mathrm{HFD}$ group after 12 and 16 weeks of diet. Oil Red-O staining confirmed steatosis in these groups. Masson's trichrome staining showed no fibrosis in any of the groups, while heart histology showed plaque formation for the apoE ${ }^{-t}$ mice with marked differences between the $\mathrm{NC}^{-}$ and the HFD diet groups. Lipid profile analysis showed a significant $(P<0.001)$ influence of mouse strain on TAG, TC, HDL, LDL levels, and a significant $(P<0.01)$ influence of diet on TC, HDL, LDL levels.

Ongoing experiments include liver TAG measurement and atherosclerotic plaque quantification. Based on our findings apoE ${ }^{-\prime-}$ animals fed a HFD for 12 weeks develop the histological features of NAFLD and will be used for our liver proteome analysis.

1. Moore JB (2010) Proc Nutr Soc 69, 211-220.

2. Tous et al. (2005) Mol Cell Biochem 268, 53-58. 